\title{
Streptococcus Intermedius Spondylodiscitis: An Unusual Presentation of Acute Back Pain in an Elderly Gentleman With Parkinson's Disease
}

\author{
Wahid Abdula, b, Amy Jones ${ }^{\mathrm{a}}$, Preeti Gupta ${ }^{\mathrm{a}}$
}

\begin{abstract}
Back pain is a common problem affecting approximately one-third of the UK adult population each year with the etiology in older people commonly attributed to osteoporosis and spondylosis. Uncommon causes, such as spondylodiscitis, can potentially cause significant complications, therefore early diagnosis and management are imperative. We present an unusual case of back pain in an elderly gentleman with Parkinson's disease (PD) attributed to Streptococcus intermedius spondylodiscitis. A 73-year-old gentleman presented with non-specific chest and abdominal pain. Inflammatory markers were elevated, consolidation demonstrated on chest X-ray, and antibiotics were commenced. Patient suffers from PD managed with selegiline, stalevo, and a self-administered apomorphine subcutaneous pump. He developed a constant severe lower back pain without neurological deficit. Streptococcus intermedius was identified on blood culture and common sources were excluded. Magnetic resonance imaging and minimal-preparation computed tomography (CT) confirmed intervertebral spondylodiscitis. A conservative approach with combination antibiotics and rehabilitation was undertaken and the patient returned to his previous functional level. We report an unusual case of spondylodiscitis in an elderly gentleman with PD. The initial presentation of radicular pain, unusual causative agent isolated, and the likely source of infection from the apomorphine pump highlight the importance of clinical suspicion, early diagnosis and management of patients with spondylodiscitis.
\end{abstract}

Keywords: Streptococcus intermedius; Spondylodiscitis; Elderly; Parkinson's disease

Manuscript accepted for publication October 18, 2013

${ }^{\mathrm{a}}$ Department of Geriatric Medicine, University Hospital Llandough, Penlan Road, Penarth, Cardiff, CF64 2XX, UK

${ }^{\mathrm{b}}$ Corresponding author: Wahid Abdul, University Hospital Llandough, Department of Geriatric Medicine, Penlan Road, Penarth, Cardiff, CF64 2XX, UK. Email: abdulw@cardiff.ac.uk

doi: http://dx.doi.org/10.4021/jmc1550w

\section{Introduction}

Back pain is a common problem affecting approximately one-third of the UK adult population each year [1]. Currently there is no convincing evidence that age affects the prevalence of back pain [2]. Etiology of back pain in older people has been attributed to osteoporosis and spondylosis [3]. However, uncommon causes such as spondylodiscitis can potentially cause significant complications, thereby early diagnosis is imperative. Despite being rare, the incidence of spondylodiscitis is increasing due to effective diagnostic techniques and increased intravenous drug usage, spinal surgery, and ageing population [4]. We present an unusual presentation of back pain in an elderly gentleman with Streptococcus intermedius spondylodiscitis.

\section{Case Report}

A 73-year-old man with known Parkinson's disease (PD) presented with a sudden onset of central chest pain preceded by a 3-week history of upper abdominal discomfort. Following investigations, biliary colic and acute coronary syndrome were excluded. His PD, diagnosed 17 years ago, was managed with selegiline, stalevo, and a self-administered subcutaneous apomorphine pump. Upon admission, he remained febrile and his inflammatory markers were elevated. A chest radiograph demonstrated left basal consolidation and intravenous co-amoxiclav was initially commenced but subsequently switched to intravenous tazocin when blood parameters failed to improve.

During inpatient stay, he continued having intermittent episodes of pyrexia. A previously described back pain, initially attributed to muscular spasm secondary to dyskinesia, had now become severe and constant with lumbar spine tenderness without neurological deficit. Plain films of the spine demonstrated lower thoracic and lumbar scoliosis. An ultrasound followed by a contrast $\mathrm{CT}$ abdomen and pelvis, initially undertaken to investigate an episode of coffee ground vomit, demonstrated severe spinal degenerative changes with mild spondylolisthesis at L4-L5. 

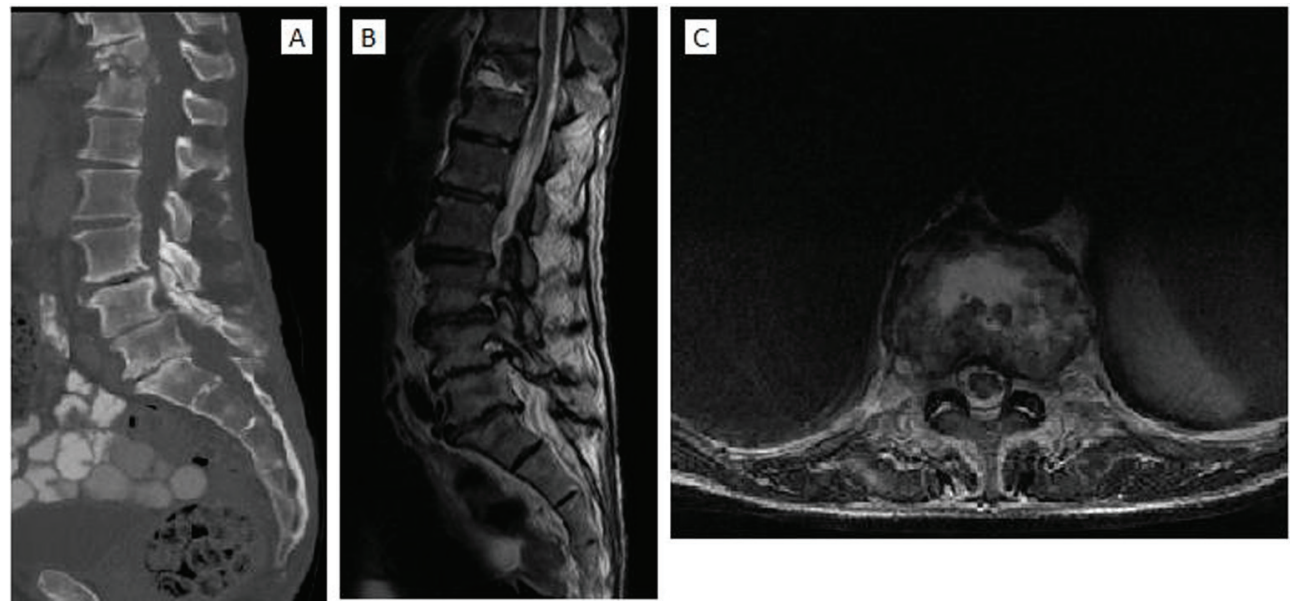

Figure 1. (A) Sagittal CT scan demonstrating destruction of T11-T12 vertebral bodies and grade 1 spondylolisthesis of L4L5. (B) Sagittal and (C) axial T2-weighted MRI scans of the thoracolumbar spine demonstrating regions of hyperintensity from T11-T12 and L4-L5 secondary to edema. There is marked destruction of intervertebral disc and vertebral column between T11-T12, an anterior epidural abscess extending from the posterior aspect of T11 and grade 1 spondylolisthesis of L4-L5 disc space.

Blood cultures obtained during admission had isolated Streptococcus intermedius. Following microbiological advice, infective endocarditis and brain abscess were excluded following transthoracic and trans-oesophageal echocardiograms and a CT brain scan. Furthermore, a minimal-preparation $\mathrm{CT}$ abdomen and pelvis was performed to exclude liver abscess, which incidentally identified an acute destructive process at T11-T12, highly suggestive of discitis. Subsequent MRI scan demonstrated vertebral body destruction, an epidural collection at T11-T12 and grade 1 spondylolisthesis, in keeping with intervertebral spondylodiscitis (Fig. 1).

Following consultation with our spinal colleagues, a conservative approach was adopted due to the potential risks of surgery in evacuating the epidural collection which was likely to be chronic and not compressing the neural elements. The patient was treated with intravenous meropenem and gentamicin for 6 weeks followed by oral antibiotics for a further 3 months. A thoracolumbar brace was recommended for stabilization of the spine, but was intolerable due to frequent dyskinesia. Within a few weeks, he improved clinically, his inflammatory markers had normalized, and he continued his rehabilitation at a community hospital achieving preadmission functional state.

\section{Discussion}

Back pain in older people is rarely associated with spondylodiscitis, with the incidence increasing due to spinal surgery, an ageing population, and increased numbers of intravenous drug users and immunocompromised patients $[4,5]$. Spondylodiscitis, a spinal infection encompassing spondylitis, discitis, and vertebral osteomyelitis, shares a bimodal age distribution peaking at less than 20 years and those aged 50 70 years and is twice more common in men [6]. Our patient was male and between 50 and 70 years of age sharing common demographics described in literature.

Due to the high frequency of back pain, insidious onset, and rarity of the disease, early diagnosis of spondylodiscitis is challenging and often completely missed.

Patients present with non-specific symptoms such as back or neck pain, occasional red flag features, and radicular pain radiating to the chest and abdomen, often leading to misdiagnosis and unnecessary investigations [7]. Initially our patient presented with radicular pain and his lower back pain was initially attributed to dyskinesia. Pyrexia is least commonly elicited by patients with spondylodiscitis, with only half of patients eliciting fever in two studies $[6,8]$. However, our patient remained febrile despite being on intravenous tazocin for his chest infection, possibly attributed to his spondylodiscitis.

In pyogenic spondylodiscitis, pathogens commonly enter hematogenously and frequently target the lumbar $(58 \%)$ and thoracic spine (30\%). In our case, spondylodiscitis had targeted the T11-T12 thoracic region. Spondylodiscitis is predominantly caused by Staphylococcus aureus [7]. In a study of 1,368 spondylodiscitis patients, the causative agent was identified in $85 \%$ of cases, with $48 \%$ attributed to Staphylococcus and only $10 \%$ to Streptococcus [9]. Gouliouris and co-workers reported a 5-20\% prevalence of streptococci species (viridans type and $\beta$-hemolytic streptococci) in pyogenic spondylodiscitis [7]. These studies demonstrate that streptococcus, the causative organism in our patient, is an uncommon cause of spondylodiscitis.

Common sources of infection for spondylodiscitis in- 
clude the genitourinary tract $(17 \%)$, infective endocarditis $(12 \%)$, and skin and soft tissue (11\%) [8]. Infective endocarditis, brain and liver abscesses were excluded in our patient. Furthermore, the self-administered subcutaneous apomorphine pump was the likely source of infection, augmented by difficult handling during dyskinesia and infrequent replacement of needles.

Elevated inflammatory markers have been reported in the majority of spondylodiscitis cases, with levels normalizing following treatment [10]. Despite intravenous tazocin, inflammatory markers remained persistently elevated in our patient. However, upon alteration to intravenous meropenem and gentamicin, inflammatory markers had normalized within a few weeks.

Due to its high sensitivity (96\%), specificity (93\%), and accuracy $(92 \%)$ in delineating anatomical structures, MRI is the modality of choice for identifying spondylodiscitis [8]. The MRI scan was diagnostic of spondylodiscitis in our patient as previous radiological investigations failed to accurately assess the anatomical structures within the spinal canal.

\section{Conclusion}

We present an unusual presentation of back pain caused by spondylodiscitis in an elderly gentleman with PD. The patient initially presented with radicular pain followed by low back pain and blood cultures isolated Streptococcus intermedius, an unlikely causative agent in pyogenic spondylodiscitis. Furthermore, we propose the likely source of infection to be the subcutaneous apomorphine pump, augmented by difficult handling due to dyskinesia and infrequent replacement of needles during self-administration. These factors highlight the importance of clinical suspicion, early diagnosis and management of patients with spondylodiscitis in order to prevent disease progression and to achieve restoration of functional activity.

\section{Conflict of Interest}

The authors declare that there is no conflict of interest.

\section{Funding}

This research received no specific grant from any funding agency in the public, commercial, or not-for-profit sectors.

\section{References}

1. Macfarlane GJ, Jones GT, Hannaford PC. Managing low back pain presenting to primary care: where do we go from here? Pain. 2006;122(3):219-222.

2. Airaksinen O, Brox JI, Cedraschi C, Hildebrandt J, Klaber-Moffett J, Kovacs F, Mannion AF, et al. Chapter 4. European guidelines for the management of chronic nonspecific low back pain. Eur Spine J. 2006;15(Suppl 2):S192-300.

3. Goel V, Young JB, Patterson CJ. Infective discitis as an uncommon but important cause of back pain in older people. Age Ageing. 2000;29(5):454-456.

4. Carragee EJ. Pyogenic vertebral osteomyelitis. J Bone Joint Surg Am. 1997;79(6):874-880.

5. Honan M, White GW, Eisenberg GM. Spontaneous infectious discitis in adults. Am J Med. 1996;100(1):8589.

6. Sapico FL, Montgomerie JZ. Pyogenic vertebral osteomyelitis: report of nine cases and review of the literature. Rev Infect Dis. 1979;1(5):754-776.

7. Gouliouris T, Aliyu SH, Brown NM. Spondylodiscitis: update on diagnosis and management. J Antimicrob Chemother. 2010;65(Suppl 3):iii11-24.

8. Mylona E, Samarkos M, Kakalou E, Fanourgiakis P, Skoutelis A. Pyogenic vertebral osteomyelitis: a systematic review of clinical characteristics. Semin Arthritis Rheum. 2009;39(1):10-17.

9. Weber M, Gubler J, Fahrer H, Crippa M, Kissling R, Boos N, Gerber H. Spondylodiscitis caused by viridans streptococci: three cases and a review of the literature. Clin Rheumatol. 1999;18(5):417-421.

10. Zarrouk V, Feydy A, Salles F, Dufour V, Guigui P, Redondo A, Fantin B. Imaging does not predict the clinical outcome of bacterial vertebral osteomyelitis. Rheumatology (Oxford). 2007;46(2):292-295. 